\title{
Avaliação do tempo de permanência como um indicador de qualidade na assistência ao paciente do serviço de urgência e emergência: uma revisão de literatura
}

\author{
Assessment of length of stay as a quality indicator in urgent and emergency care patient care: a
}

literature review

Evaluación de la estancia hospitalaria como indicador de calidad en la atención al paciente de urgencias y urgencias: revisión de la literatura

Recebido: 24/11/2021 | Revisado: 30/11/2021 | Aceito: 01/12/2021 | Publicado: 01/12/2021

\author{
Francisca Luana Vieira Barroso \\ ORCID: https://orcid.org/0000-0002-1953-5174 \\ Centro Universitário da Amazônia, Brasil \\ E-mail: luhbarroso83@gmail.com \\ Josivandra Luiane Pimenta da Rocha \\ ORCID: https://orcid.org/0000-0002-7660-1115 \\ Centro Universitário da Amazônia, Brasil \\ E-mail: josivandra.pimentas2@gmail.com \\ Francisca Farias Cavalcante \\ ORCID: https://orcid.org/0000-0003-0827-4340 \\ Centro Universitário da Amazônia, Brasil \\ E-mail: enfafrancavalcante2@gmail.com
}

\begin{abstract}
Resumo
Os protocolos de atendimento ao usuário, norteados pelos princípios da Política de Humanização (PNH), trouxeram aos gestores e usuários uma nova concepção de assistência no SUS, a satisfação do usuário sobre aos cuidados prestados, foi considerado um indicador de qualidade. Então todos os serviços de saúde passaram a trabalhar com a PNH, contemplando também os serviços de urgência e emergência. Discorrer sobre a avaliação do tempo de espera como indicador de qualidade do serviço de urgência, abordar implantadas, correlacionar os achados com a realidade do serviço apresentado nas literaturas. Caráter descritivo, revisão de literatura, utilizando artigos de periódicos científicos da área da saúde, teses e dissertações, disponíveis em: SCIELO, LILACS, MEDLINE; nos idiomas português, inglês e espanhol. Analisados pelo método de Bardin. Na atenção de urgência e emergência, pronto atendimento, assistência pré-hospitalar e outros; as diretrizes a serem seguidas são: acolher a demanda pela avaliação de risco, garantir o acesso referenciado, aumentar a resolutividade, prover o acesso à estrutura hospitalar e a transferência segura, caso seja necessário. O tempo de espera em casos de emergência é crucial para o desfecho do paciente e não pode ser protelado, podendo ser mitigado através da aplicação de protocolos organizativos. Destaca -se também que todas as fases pro processo de ACCR devem ser seguidas, pois falhas em um ponto do processo, podem acarretas em desfechos como superlotação da unidade, agravamento de quadros clínicos dos pacientes ainda em sala de espera e sobrecarga de profissionais.
\end{abstract}

Palavras-chave: Indicadores da qualidade em assistência à saúde, Assistência de urgência, Serviço de urgência.

\begin{abstract}
User service protocols, guided by the principles of the Humanization Policy (PNH), brought to managers and users a new concept of care in the SUS, user satisfaction with the care provided, was considered a quality indicator. So all health services started to work with the PNH, also including urgent and emergency services. Discuss the assessment of waiting time as an indicator of quality of the emergency service, address implemented, correlate the findings with the reality of the service presented in the literature. Descriptive character, literature review, using articles from scientific journals in the health area, theses and dissertations, available in: SCIELO, LILACS, MEDLINE; in Portuguese, English and Spanish. Analyzed by the method of Bardin. In urgent and emergency care, emergency care, pre-hospital care and others; the guidelines to be followed are: accepting the demand for risk assessment, guaranteeing referenced access, increasing resoluteness, providing access to the hospital structure and safe transfer, if necessary. Waiting time in emergency cases is crucial for the patient's outcome and cannot be delayed, and can be mitigated through the application of organizational protocols. It is also noteworthy that all phases of the ACCR process must be followed, as failures at one point in the process can lead to outcomes such as overcrowding in the unit, worsening of clinical conditions of patients still in the waiting room and overload of professionals.
\end{abstract}

Keywords: Quality indicators in health care; Emergency assistance; Emergency service. 


\begin{abstract}
Resumen
Los protocolos de atención al usuario, guiados por los principios de la Política de Humanización (PNH), llevaron a los gestores y usuarios un nuevo concepto de atención en el SUS, la satisfacción del usuario con la atención brindada, fue considerada un indicador de calidad. Entonces todos los servicios de salud comenzaron a trabajar con la PNH, incluyendo también los servicios de urgencia y emergencia. Discutir la valoración del tiempo de espera como indicador de la calidad del servicio de emergencia, dirección implementada, correlacionar los hallazgos con la realidad del servicio presentado en la literatura. Carácter descriptivo, revisión de la literatura, utilizando artículos de revistas científicas del área de la salud, tesis y disertaciones, disponibles en: SCIELO, LILACS, MEDLINE; en portugués, inglés y español. Analizado por el método de Bardin. En atención de urgencia y emergencia, atención de emergencia, atención prehospitalaria y otros; las pautas a seguir son: aceptar la demanda de evaluación de riesgos, garantizar el acceso referenciado, aumentar la resolución, brindar acceso a la estructura hospitalaria y traslado seguro, si es necesario. El tiempo de espera en casos de emergencia es crucial para el resultado del paciente y no se puede retrasar, y se puede mitigar mediante la aplicación de protocolos organizativos. También es de destacar que se deben seguir todas las fases del proceso ACCR, ya que las fallas en un punto del proceso pueden llevar a resultados como hacinamiento en la unidad, empeoramiento de las condiciones clínicas de los pacientes que aún se encuentran en la sala de espera y sobrecarga de Profesionales.
\end{abstract}

Palabras clave: Indicadores de calidad en la asistencia sanitaria; Asistencia de emergencia; Servicio de emergencia.

\title{
1. Introdução
}

Em busca de uma melhor assistência nos serviços hospitalares, o Ministério da Saúde brasileiro lançou o Programa Nacional de Humanização da Assistência Hospitalar (PNHH), o que contribuiu para o alargamento da abrangência dos horizontes da assistência humanizada em saúde, pois em 2003 houve a criação da Política Nacional de Humanização (PNH) Humaniza SUS. Este eixo norteador da gestão e da assistência em saúde, tem como fundamento a responsabilização conjunta dos sujeitos envolvidos nos diversos elos da assistência, ou seja, a visão de humanização da assistência na saúde passou a ser trabalhada desde a estrutura do serviço até os profissionais que prestam os cuidados, gerando indicadores de qualidade no serviço de saúde (Sousa et al., 2019).

Em sua obra, Chiavenato (2003), destacou sistema de Gestão da Qualidade Total (GQT), desde então as instituições de saúde têm implantado esse sistema em seus serviços, e definindo um processo que é capaz de detectar as necessidades de seus usuários; em suas experiências os autores Balsanelli e Jericó (2005) ressaltam que tal implementação, aponta para melhorias nas prestações de serviços.

Desse modo, a elaboração de protocolos de atendimento ao usuário, norteados pelos princípios da $\mathrm{PNH}$, trouxe à vista dos gestores e dos usuários uma nova concepção de assistência no âmbito do SUS, onde a satisfação do usuário em relação aos cuidados prestados, passou a ser considerado um indicador de qualidade.

Desde então todos os serviços de públicos de saúde passaram a trabalhar sob a ótica da humanização, em todos os níveis de assistência, contemplando também os serviços de urgência e emergência em todas as suas particularidades.

Para melhor entendimento do tema desta obra, se faz necessário reconhecer o conceito o seguinte conceito, Brasil (2014) define urgência, como sendo uma situação imprevista de agravo à saúde que incorra ou não em risco de morte, cujo portador necessita de assistência imediata.

Silva; Matsuda e Waidman (2012), ressaltam que no momento em que se depara com a saúde em ameaça, o ser humano apresenta alguns sentimentos, dentre eles estão: medo, angústia, incerteza, ansiedade. Situações essas que fazem parte do cotidiano dos serviços de urgência. $\mathrm{E}$ os profissionais precisam estar preparados para lidar com essa clientela, diminuindo o tempo de espera afim de amenizar o desconforto, prestando assistência de qualidade.

Além do relacionamento interpessoal, outros fatores são determinantes para a avaliação da qualidade dos serviços de saúde. Nesta obra será destacada avaliação do tempo de espera como um fato de qualidade na assistência de urgência e emergência. 
Diante do exposto, esta obra tem por objetivo discorrer sobre a avaliação do tempo de espera como indicador de qualidade do serviço de urgência, abordando diretrizes e protocolos de já implantados, correlacionando os achados com a realidade do serviço apresentado nas literaturas.

\section{Metodologia}

Este é um estudo de caráter descritivo, do tipo revisão de literatura, para o qual foram utilizados artigos de periódicos científicos da área da saúde, manuais instrutivos do governo brasileiro e protocolos de instituições de saúde disponíveis à comunidade, assim como teses e dissertações, disponíveis nas seguintes bases de dados científicos: SCIELO (Scientific Eletronic Library Online), LILACS (Literatura Latino - Americana e do Caribe em Ciências da Saúde), BVS (Biblioteca virtual da Saúde), MEDLINE (Medical Literature Analysis and Retrieval System Online); nos idiomas português, inglês e espanhol; usando como descritores: Indicadores da Qualidade em Assistência à Saúde, Assistência de Urgência, Serviço de Urgência.

Para Gerhart e Silveira (2009), o estudo descritivo tem o objetivo de descrever características, servindo de suporte para a análise de características, sejam elas populacionais, características de um dado fenômeno, aspectos de documentos ou ainda experiências, afim de se obter um estudo mais completo. Dessa forma o estudo descritivo se constitui uma das classificações das pesquisas científicas.

Quanto aos critérios de inclusão: ser escrito nos idiomas anteriormente citados, ter relação com o tema, ter sido publicados entre os anos de 2003 e 2021; teve como critérios de exclusão: textos incompletos, versão apenas em resumos, textos sem referência à autoria e não atender aos critérios de inclusão.

Para o processo de análise do conjunto de dados obtidos, foi usado como referência a obra de Laurence Bardin, que é a literatura de referência atualmente em análise de conteúdo. De acordo com Bardin (2012), a análise de conteúdo é um conjunto de técnicas de análise de comunicações, que se utiliza de procedimentos sistemáticos e objetivos de descrição do conteúdo das mensagens. As fases de análises propostas pela autora são respectivamente: pré - análise, exploração sistemática dos documentos e tratamento dos resultados. Os passos estão descritos na Figura 1.

Figura 1. Síntese do método de análise de conteúdo de Bardin.

\section{MÉTODO DE ANÁLISE DE CONTEÚDO}

\begin{tabular}{c|l}
\hline $\begin{array}{c}\text { FASE I } \\
\text { Pré - análise }\end{array}$ & $\begin{array}{l}\text { Seleção dos materiais que tem relação com o objeto de estudo, embasamento argumentativos } \\
\text { para os resultados obtidos. Nesta fase são aplicados os critérios de inclusão e exclusão, para } \\
\text { facilitar a obtenção de dados que serão realmente aproveitados. }\end{array}$ \\
\hline $\begin{array}{c}\text { FASE II } \\
\text { Exploração do } \\
\text { material }\end{array}$ & $\begin{array}{l}\text { Estudo e leitura minuciosa dos materiais obtidos. Nesta fase são avaliação os escritos e } \\
\text { selecionados os que serão necessários para sustentar do tema de estudo, assim como os que } \\
\text { serão utilizados para fazer correlação com a proposta do tema em questão. }\end{array}$ \\
\hline $\begin{array}{c}\text { FASE III } \\
\text { Tratamento dos } \\
\text { resultados }\end{array}$ & $\begin{array}{l}\text { Validação dos dados analisados. Relação dos objetivos com os resultados, e possível } \\
\text { surgimento de novas hipóteses. }\end{array}$ \\
\hline
\end{tabular}

Fonte: Azevedo et al. (2021).

\section{Resultados e Discussão}

Ao todo foram encontrados 30 artigos no período de 2003 a 2021, utilizando os critérios de inclusão e exclusão, após aplicação da análise de conteúdo, proposta por Bardin (2012), restaram apenas 11 artigos elegíveis (Quadro 1). Que serão discutidos nesta sessão. 
Quadro 1. Artigos Elegíveis para o estudo.

\begin{tabular}{|c|c|c|}
\hline AUTOR & ANO & TÍTULO \\
\hline Bittencourt, e Hortale & 2009 & $\begin{array}{l}\text { Intervenções para solucionar a superlotação nos serviços de } \\
\text { emergência hospitalar: uma revisão sistemática. }\end{array}$ \\
\hline Hermida et al. & 2018 & $\begin{array}{l}\text { User embracement with risk classification in an emergency } \\
\text { care unit: an evaluative study }\end{array}$ \\
\hline Lopes, A. B. A. & 2019. & $\begin{array}{c}\text { Percepção do usuário do SUS, no atendimento de urgência } \\
\text { e emergência do hospital municipal de Itaituba }\end{array}$ \\
\hline Lopes, J. B. & 2011 & $\begin{array}{c}\text { Enfermeiro na classificação de risco em serviços de } \\
\text { emergência: revisão integrativa }\end{array}$ \\
\hline Mendes, A. C. G. & 2009 & $\begin{array}{l}\text { Avaliação da Qualidade da Assistência de Urgência e } \\
\text { Emergência: Uma abordagem por triangulação de métodos. }\end{array}$ \\
\hline Paula et al. & 2019 & $\begin{array}{l}\text { Humanization of care: reception and screening in risk } \\
\text { classification }\end{array}$ \\
\hline Prudêncio et al. & 2016 & $\begin{array}{l}\text { Percepção de enfermeira(o)s sobre acolhimento com } \\
\text { classificação de risco no serviço de pronto atendimento }\end{array}$ \\
\hline Silva et al. (a) & 2020 & $\begin{array}{c}\text { Humanização da assistência: acolhimento e classificação de } \\
\text { risco no serviço de urgência e emergência }\end{array}$ \\
\hline Silva et al. (b) & 2016 & Triage in an adult emergency service: patient satisfaction \\
\hline Sousa et al. & 2019 & $\begin{array}{l}\text { Humanização nos serviços de urgência e emergência: } \\
\text { contribuições para o cuidado de enfermagem }\end{array}$ \\
\hline Valentina e Santos & 2013 & Políticas de saúde em emergência e a enfermagem \\
\hline
\end{tabular}

Fonte: Barroso, Rocha e Cavalcante (2021).

Brasil (2003a) destaca que O sistema integral de atenção às urgências deve abranger desde a atenção primária, perpassando pela terapia intensiva e reabilitação, afim de envolver todo o manejo das urgências coletivas. Diante disso, é possível inferir que o atendimento de urgência não se limita às unidades de pronto socorro, mas se alarga à toda a rede de atenção à saúde, estando todos os níveis desta rede, sob as diretrizes da Rede de Atenção de Urgência e Emergência (RUE).

A RUE é rede complexa e que atende a diferentes condições [...] é composta por diferentes pontos de atenção, de forma a dar conta das diversas ações necessárias ao atendimento às situações de urgência. Desse modo, é necessário que seus componentes atuem de forma [...] sinérgica. Além disso, de forma transversal a todos os componentes, devem estar presentes o acolhimento, a qualificação profissional, a informação e a regulação de acesso (Brasil, 2013b). (Com ênfase acrescentada pela autora)

Dentre as diretrizes da Rede de Atenção de Urgência e Emergência, destacam se:

I. Universalidade, equidade e integralidade da atenção as situações de urgência e emergência, incluindo as clínicas, gineco-obstétricas, psiquiátricas, pediátricas e as relacionadas às causas externas (traumatismos, acidentes e violência);

II. Ampliação do acesso com acolhimento, aos casos agudos e em todos os pontos da atenção;

III. Classificação de risco;

IV. Humanização da atenção.

Brasil (2003d), estabeleceu alguns indicadores de qualidade com o objetivo de estabelecer metodologias para avaliação das unidades a partir de dados coletados no processo de atendimento, isto se deu pela necessidade de se levar em 
consideração os serviços de saúde, como sendo elementos em uma rede interligada, um sistema. Dentre os tais marcadores, se destaca o tempo médio de espera e permanência no serviço (por parte do cliente) e o tempo médio de resposta (do serviço de urgência).

A busca pela criação de diretrizes e protocolos para o atendimento nos serviços de urgência demonstram a preocupação em organizar o atendimento ao usuário, doutro modo cada instituição deveria ter sua forma organizativa. A padronização da organização do cuidado é sempre pautada na qualidade desde o acolhimento ao paciente (Brasil, 2013b). Pode - se observar essa vigilância nos componentes da RUE descritos na Figura 2.

Figura 2. Componentes da rue e suas interfaces.

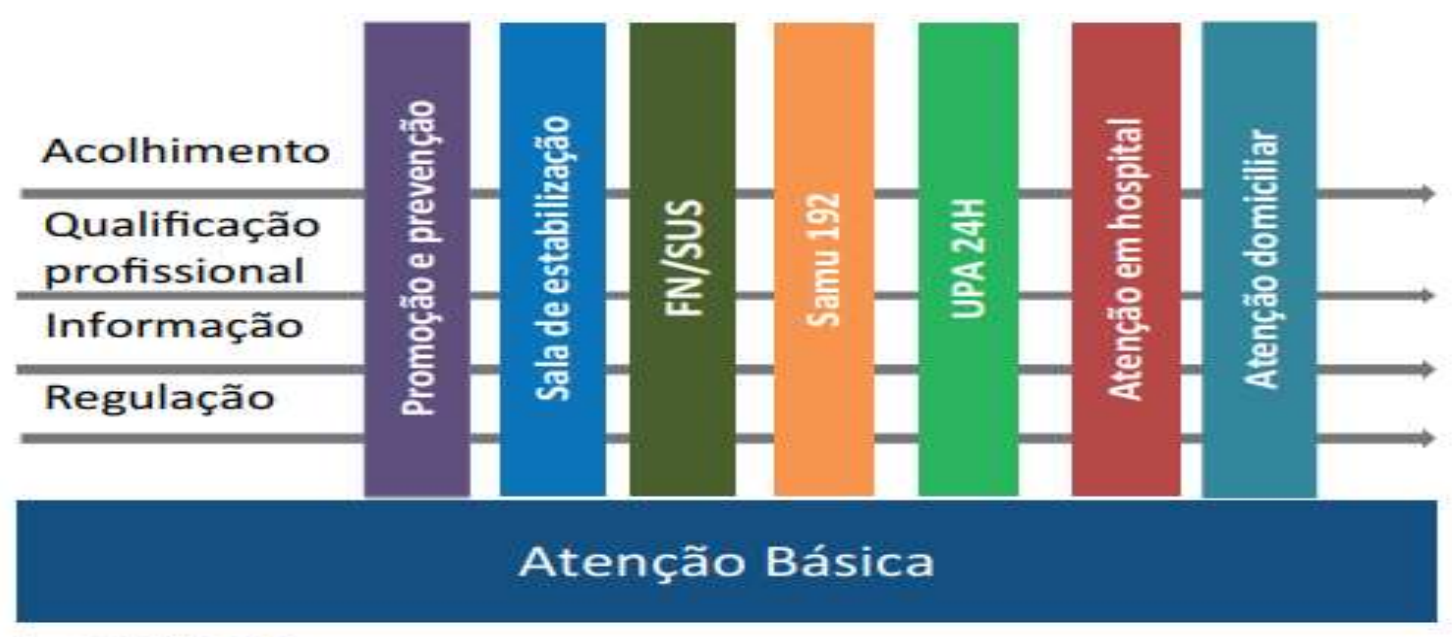

Fonte: SAS/MS, 2011.

Fonte: Brasil (2013b).

Brasil (2013b) destaca que: na atenção de urgência e emergência, pronto atendimento, assistência pré-hospitalar e outros; as diretrizes a serem seguidas são:

a) Acolher a demanda pela avaliação de risco, garantir o acesso referenciado aos demais níveis de assistência;

b) Atentar para a referência e a contra referência;

c) Aumentar a resolução da urgência e emergência;

d) Prover o acesso à estrutura hospitalar e a transferência segura, caso seja necessário.

As diretrizes acima citadas, se constituem pontos categóricos e de alta influência sob o tempo de espera do paciente; Lopes (2009) revalida a fala anterior quando diz que o tempo de espera em casos de emergência é crucial para o desfecho do paciente e não pode ser protelado. O acolhimento com classificação de risco - ACCR é um recurso muito importante para o reconhecimento de pacientes que precisam de atendimento imediato, considerando os riscos e prejuízos à saúde ou intensidade de sofrimento, devendo o profissional enfermeiro, priorizar de acordo com a gravidade clínica do paciente, e não por ordem de chegada ao serviço (Brasil, 2014c).

Brasil (2009) ainda destaca que o protocolo de ACCR é um instrumento necessário e útil, contudo ainda não é o suficiente, já que não é capaz de abranger aspectos subjetivos, sociais e culturais, cuja a compreensão é de fundamental importância para a efetividade da avaliação do risco e da vulnerabilidade de cada usuário que procura a unidade de urgência. Ressalta também, que o protocolo faz substituição da interação, do diálogo, da escuta, e do respeito, em suma, do acolhimento do paciente e de sua queixa, para avaliação do seu potencial de agravamento.

O ACCR vem sendo utilizada em diversos países; com isso, foram desenvolvidos diversos protocolos, com o objetivo de diminuir o tempo de espera e atender às necessidades do usuário, que precisa de assistência imediata. Por isso, todos eles 
são baseados na avaliação inicial do paciente, já bem desenvolvida para o atendimento às situações de catástrofes e adaptada para os serviços de urgência (Brasil, 2009e).

No Brasil, o Acolhimento com Classificação de Riscos (ACCR), foi proposta pelo Ministério da Saúde, através da PNH. Em seu estudo, Hermida (2018) destaca que a utilização de protocolos de ACCR é uma intervenção com potencial decisivo para a reorganização do atendimento nos serviços de urgência e implementação da produção da saúde. De acordo com Brasil (2009), os critérios utilizados são:

1. A classificação de risco é atividade realizada por profissional de enfermagem de nível superior, preferencialmente com experiência em serviço de urgência, e após capacitação específica para a atividade proposta;

2. Recomenda-se que o protocolo tenha no mínimo quatro níveis de classificação de risco;

3. Recomenda-se o uso preferencial de cores, e não de números, para a classificação de risco (exemplo no caso de quatro níveis de classificação, do mais grave ao menos grave: vermelho, amarelo, verde, azul).

Silva et al. (2020a) destaca que, nesse contexto, o acolhimento pode ser realizado por qualquer profissional desde que este esteja devidamente treinado, enquanto a Classificação de Risco é de responsabilidade do profissional enfermeiro. A participação ativa de toda a equipe multiprofissional é muito necessária para o sucesso do ACCR, porém, os profissionais de enfermagem exercem um papel de fundamental importância nesse processo. Cabe ao enfermeiro a classificação de risco, e, são os trabalhadores da enfermagem quem comumente mantém contato direto e contínuo com os pacientes.

O instrumento para Classificação de Risco (CR) que vem sendo utilizado nos serviços públicos de urgência é o protocolo Manchester Triage System - MTS@ é uma metodologia de trabalho desenvolvida na Inglaterra, na década de 1990. Em sua obra, Silva et al. (2016b) ressalta a utilização do protocolo de Manchester sendo utilizado em muitos países e adaptado para a realidade brasileira; destaca ainda que o objetivo desse instrumento consiste em classificar os usuários de acordo com a prioridade de intervenção.

Figura 3. Ilustração resumo da classificação de risco de acordo com o protocolo de Manchester Triage System - MTS@.

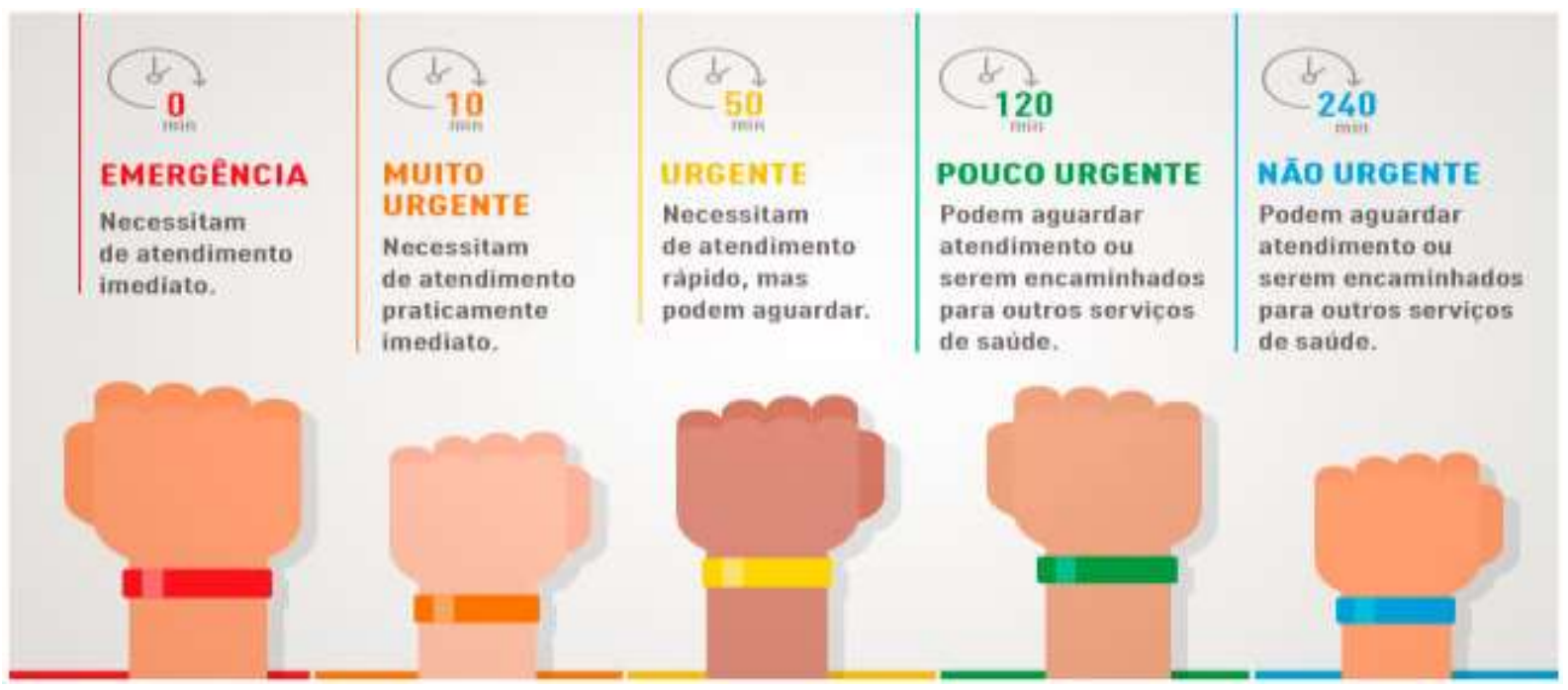

Fonte: Rede Educação (2020).

Para isso o método segue a seguinte sequência: identificar a queixa inicial do paciente, seguimento do fluxograma de decisão e, por fim, estabelecimento do tempo de espera de acordo com a gravidade. O fluxograma estabelece a classificação de atendimento de acordo com um sistema de cores como descrito na Figura 3 (Silva et al., 2016b). 
Além da CR, a contrarreferência foi citada como um fator a ser levado em consideração, pois o número elevado de pacientes em quadro "não urgentes" aumenta o tempo de espera e consequentemente contribui para a insatisfação do cliente; tal avaliação aponta para a necessidade de um melhor preparo, assim como uma melhor comunicação entre os dos profissionais, além da constante reavaliação das pessoas em espera por atendimento, pois o quadro clínico pode mudar rapidamente (Hermida et al., 2018). Os autores anteriormente citados corroboram que a elaboração de protocolos pactuados pelos gestores é capaz de garantir a execução efetiva da contrarreferência.

No que se refere a mitigação do tempo de espera dos usuários, Servin et al. (2009) e Prudêncio et al. (2016), corroboram que definir protocolos clínicos, com o objetivo de garantir a eliminação de intervenções desnecessárias e respeitar as diferenças e as necessidades do sujeito, são fundamentais para esse processo.

Em uma análise retrospectiva, é possível inferir que diante de tantos esforços empregados para melhorar a qualidade do atendimento dos serviços de urgência, as experiências de diversos autores demonstram que na prática, ainda se tem um longo caminho para se chegar ao objetivo almejado, principalmente no que tange aos serviços públicos de urgência. Fatores como a falta de recursos humanos e materiais se destacam na avaliação negativa do tempo de espera como fator de qualidade no serviço de urgência (Mendes, 2009; Valentina, 2013; Lopes, 2019).

Lopes (2019) descreveu a insatisfação dos usuários com os serviços de uma unidade pública de urgência; em sua experiência apontou, além do tempo de espera, a estrutura e tratamento recebido por parte dos profissionais como ponto negativo na avaliação da qualidade da assistência prestada. Bittencourt e Hortale (2009), apontam que a realidade dos setores de urgência públicos mostra a superlotação diária que sobrecarrega o setor e os profissionais. Isto se dá tanto por falta de mais unidades de atendimento, quando por conta do quantitativo de profissionais disponíveis em serviço (Bittencourt e Hortale, 2009; Paula, Ribeiro e Werneck, 2019).

Em contra ponto, em um estudo semelhante conduzido por Silva et al. (2016b), apresentou um grau elevado de satisfação do usuário com a assistência prestada, neste estudo foram utilizadas as seguintes variáveis para mensurar o grau de satisfação: tempo de espera, equipe de saúde (nos seguintes aspectos: educação, respeito interesse e confiança), suporte oferecido pelo serviço. Na oportunidade foi observado que os índices de satisfação foram superiores a $85 \%$ para satisfeito e muito satisfeito em relação ao tempo de espera, e sobre a equipe de saúde foram superiores a $96 \%$ para satisfeito e muito satisfeito.

Diante do exposto deve se ressaltar que a estrutura do ambiente e os profissionais envolvidos na assistência são capazes de amenizar a insatisfação dos usuários, Silva et al. (2016b) concluem que as informações sobre o tempo de espera corretamente sinalizada e informada, e a reavaliação constante do quadro do paciente em espera do atendimento, diminuem a insatisfação e contribuem para uma assistência de fato Humanizada.

Para se poder fazer a avaliação da humanização da assistência é necessário que o usuário saiba definir corretamente o que e o que não é, uma assistência humanizada. Ao avaliar, o usuário deve observar não somente a forma que é acolhido no setor, mas também a estrutura na qual ele é atendido, se a sua demanda foi atendida, se foi em tempo hábil se foi realizada da maneira adequada.

Barros e Sousa (2016), Filho e Garbin (2010), reforçam que o acesso à informação correta a respeito dos direitos e deveres do usuário, são de primordial importância para participação popular na avaliação da qualidade da assistência.

\section{Considerações Finais}

Em todas os critérios de avaliação, que seja do serviço de urgência, quer seja da classificação de risco ou da visão geral do atendimento, sempre se destaca o tempo de permanência e espera como um fator primordial. Fator este que por muitas vezes apresenta altos níveis de insatisfação por parte do usuário. 
Fatores como a falta de estrutura física, falta de insumos, ou menos de profissionais devidamente capacitados para realizar a assistência de maneira adequada, se constitui um entrave nesse processo. A utilização de protocolos e instrumentos organizativos, só será eficaz se o profissional que o está aplicando for adequadamente treinado para tal função.

Destaca -se também que todas as fases pro processo de ACCR devem ser seguidas, pois falhas em um ponto do processo, podem acarretas em desfechos como superlotação da unidade, agravamento de quadros clínicos dos pacientes ainda em sala de espera e sobrecarga de profissionais.

A elaboração e a análise do fluxograma de atendimento no pronto-socorro, é indicada para identificar os pontos onde se concentram os problemas, eles promovem uma reflexão profunda sobre o processo de trabalho.

Diante disso surge a necessidade da educação permanente dentro dos serviços de urgência aos profissionais envolvidos na assistência, para que esta seja melhor organizada, oferecendo ao usuário uma assistência humanizada e integral de forma resolutiva, acolhedora. Em busca de constantes melhorias em prol da qualidade da assistência de saúde, sugere -se a realização de estudos que avaliem o conhecimento e o engajamento da equipe de saúde para a qualidade dos serviços em ambientes de urgência e emergência.

\section{Referências}

Azevedo, D. K. L., Silva, C. M. P., \& Maia, A. L. (2021). O papel da gestão de enfermagem na implementação da meta de cirurgia segura: uma revisão de literatura. Research, Society and Development, 10(14), e584101422711, 2021 (CC BY 4.0) | ISSN 2525-3409 | DOI: http://dx.doi.org/10.33448/rsdv10i14.22711.

Balsanelli, A. P. E., \& Jericó, M. De C. (2005). Os reflexos da gestão pela qualidade total em instituições hospitalares brasileiras. Acta Paulista Enfermagem. 2005;18(4):397-402. https://www.scielo.br/pdf/ape/v18n4/a08v18n4.pdf.

Bardin, L. Análise de conteúdo: a revisão de Laurence Bardin. (2012). Revista Eletrônica de Educação. Programa de Pós-graduação em Educação. 6(1), mai. 2012. http://dx.doi.org/10.14244/\%2519827199291.

Barros, F. P. C., \& Sousa, M. F. (2016). Equidade: Seus conceitos, significações e implicações para o SUS. Revista Saúde e Sociedade, São Paulo, 25(1), p. 918, 2016. ISSN: 1984-0470. http://www.revistas.usp.br/ sausoc/issue/view/8534.

Bittencourt, R. J., \& Hortale, V.A. (2009). Intervenções para solucionar a superlotação nos serviços de emergência hospitalar: uma revisão sistemática. Caderno de Saúde Publica. 2009;25(7):1439-1454. doi:10.1590/S0102-311X2009000700002.

Brasil. (2003a) Ministério da Saúde. Humaniza SUS - Política Nacional de Humanização: A humanização como eixo norteador das práticas de atenção e gestão em todas as instâncias do SUS.). Brasília, 2003. (Série B. Básicos de Saúde).

Brasil. (2003e). Qualidade nos Serviços de Urgência. Alguns Indicadores. Ministério da saúde secretaria de atenção à saude departamento de atenção especializada coordenação geral de urgência/emergência. Seminário da Política Nacional de Atenção Integral às Urgências. 2003.

Brasil. (2009d). Ministério da Saúde. Política Nacional de Humanização da Atenção e Gestão do SUS. Acolhimento e classificação de risco nos serviços de urgência. Brasília: MS; 2009. Secretaria de Atenção à Saúde. https://www.gov.br/saude/pt-br/acesso-a-informacao/acoes-e-programas/politica-nacional-dehumanizacao-humanizasus\#: :text=A\%20Pol\%C3\%ADtica\%20Nacional\%20de\%20Humaniza\%C3\%A7\%C3\%A3o,entre\%20 gestores $\% 2 \mathrm{C} \% 20$ trabalhadores $\% 20 \mathrm{e} \% 20 \mathrm{usu} \% \mathrm{C} 3 \% \mathrm{~A} 1$ rios.

Brasil. (2013b). Ministério da saúde. Manual Instrutivo da Rede de Atenção às Urgências e Emergências no Sistema Único de Saúde (SUS) - RUE. Secretaria de Atenção à Saúde. Departamento de Atenção Especializada 2013. https://bvsms.saude.gov.br/bvs/publicacoes/manual_instr utivo_rede_atencao_urgencias.pdf

Brasil. (2014c). Ministério da Saúde. Boas Práticas para Organização e Funcionamento de Serviços de Urgência e Emergência. PORTARIA N 354 , DE 10 DE MARÇO DE 2014. http://bvsms.saude.gov.br/bvs/saudelegis /gm/2014/prt0354_10_03_2014.html.

Chiavenato, I. (2003). Introdução à teoria geral da administração. $7^{a}$ edição, Rio de Janeiro: Elsevier, 2003. 634p.

Filho, A. C. P., \& Garbin, C. A. S. (2010). A saúde e seu direito sob o olhar do usuário. Revista Brasileira de Pesquisa em Saúde 2010; $12(3):$ 39-45.

Gerhardt, T. E., \& Silveira, D. T. (2009). Métodos de pesquisa. Planejamento e gestão para o desenvolvimento rural da SEAD/UFRGS. Universidade Federal do Rio Grande do Sul. https://lume.ufrgs.br/bitstream/handle/10183/52806/000728684.pdf?sequence=1\&isAllowed=y.

Hermida, P. M. V., Nascimento, E. R. P., Echevarría-Guanilo, M. E., Brüggemann, O. M., \& Malfussi, L. B. H. (2018). User embracement with risk classification in an emergency care unit: an evaluative study. Revista da Escola de Enfermagem da USP. 2018;52:e03318. DOI: http://dx.doi.org/10.1590 /S1980-220X2017001303318.

Lopes, A. B. A. (2019a). Percepção do usuário do SUS, no atendimento de urgência e emergência do hospital municipal de Itaituba. TESE. Centro de estudos superiores de itaituba .- FAI. Curso de bacharelado em enfermagem. Pará, Brasil. 2019. http://www.faculdadedeitaituba.com.br/tcc/a-percepcao-do-usuariodo-sus--no-atendimento-de-urgencia-e-emergencia-do-hospital-municipal-de-itaituba--para--brasil 
Research, Society and Development, v. 10, n. 15, e595101523820, 2021

(CC BY 4.0) | ISSN 2525-3409 | DOI: http://dx.doi.org/10.33448/rsd-v10i15.23820

Lopes, J. B. (2011b). Enfermeiro na classificação de risco em serviços de emergência: revisão integrativa. 2011. Monografia (Bacharel em Enfermagem) Universidade Federal do Rio Grande do Sul, Porto Alegre/RS. https://www.lume.ufrgs.br/handle/10183/37529.

Mendes, A. C. G. (2009). Avaliação da Qualidade da Assistência de Urgência e Emergência: Uma abordagem por triangulação de métodos. 2009. TESE. Doutorado em Saúde Pública. Fundação Oswaldo Cruz - centro de pesquisas Aggeu Magalhães. Recife, Brasil. https://www.cpqam.fiocruz. br/bibpdf/2009mendes-acg.pdf.

Paula, C. F. B., Ribeiro, R. C. H. M., \& Werneck, A. L. (2019). Humanization of care: reception and screening in risk classification. Journal of Nursing UFPE on line, v. 13, n. 4, p. 997-1005, apr. 2019.

Prudêncio, C. P. G., Monteiro, R. A. N., Ribeiro, B. C. M., Gomes, M. S. M., \& Manhães, L. S. P. (2016). Percepção de enfermeira(o)s sobre acolhimento com classificação de risco no serviço de pronto atendimento. Revista Baiana de Enfermagem, Salvador, 30(2), p. 1-10, abr./ jun. 2016. DOI: https://doi.org/10.18471/rbe.v30i2.14917.

Servin, S. C. N., Pinheiro, E., Maciel, D. O., Neto, A. S., Matos, R. M., Brito, L. C. V., Portela, M. A., Belfort, J. F., Cabral, L. L. S., Meneses, M. R. R., Araujo, D. M. M., Melo, M. R., Baldez, C. F., \& Lima, F. (2009). Protocolo de acolhimento com classificação de risco, Política Municipal de humanização, 2009. https://bvsms.saude.gov.br/bvs/publicacoes/protocolo_acolhimento_classificacao_risco.pdf.

Silva, M. P. B., Negreiros, I. G. R., Souza, D. V., Frota, M. M. C., Baldoino, A. C. S., Máximo, L. W. M., Machado, B. A. S., Alencar, V. P., Sampaio, A. S., França, D. C., Brito, R. S., \& Fagundes, G. R. S. (2020a). Humanização da assistência: acolhimento e classificação de risco no serviço de urgência e emergência. Revista Política, planejamento e gestão em saúde, n.6. Ponta Grossa, PR: Atena, 2020. DOI: 10.22533/at.ed.26220270816.

Silva, P. L., Paiva, L., Faria, V. B., Ohl, R. I. B., \& Chavaglia, S. R. R. (2016b). Triage in an adult emergency service: patient satisfaction. Revista da Escola de Enfermagem USP. 2016, 50(3), p. 427-432. DOI: http://dx.doi.org/10.1590/S0080-623420160000400008.

Sousa, K. H. J. F., Damasceno, C. K. C. S., Almeida, C. A. P. L., Magalhães, J. M., \& Ferreira, M. A. (2019). Humanização nos serviços de urgência e emergência: contribuições para o cuidado de enfermagem. Revista Gaúcha de Enfermagem, Porto Alegre, v. 40, e20180263, 2019. DOI: https://doi.org/10.1590/1983-1447.2019.20180263

Valentina, M. R. S., \& Santos, M. L. S. C. (2013). Políticas de saúde em emergência e a enfermagem. Revista de Enfermagem da UERJ. Rio de Janeiro/RJ, 17(2), 2013. 